\title{
The clinical character of hand-foot-and-mouth-disease (HFMD) with neurological manifestation: A report of 22 death and 36 recovered cases.
}

\author{
Fuyong Jiao' ${ }^{1}$, Huiling Deng ${ }^{2}$, Fiaz Ahmad ${ }^{*}$, Yang Zhao ${ }^{1}$, Zhilong Mu${ }^{1}$, Haider-Shamsi Shua ${ }^{4}$, \\ Xiaofang $\mathrm{Wu}^{5}$, Tingyuan $\mathrm{Gu}^{6}$ \\ ${ }^{1}$ Shaanxi Provincial People's Hospital, Xi'an, P.R. China \\ ${ }^{2}$ Xi' an Children's Hospital, Xi'an, P.R China \\ ${ }^{3}$ Key Laboratory for Space Bioscience and Biotechnology, School of Life Sciences, North-Western Polytechnical \\ University, Xi'an, Shaanxi, P.R China \\ ${ }^{4}$ Shenmu Hospital, Yulin, P.R China \\ ${ }^{5}$ Western and Northern Women and Children's Hospital, Xi'an, P.R China \\ ${ }^{6}$ Clinical Pediatric Class 1401 of Xi'an Medical University, Xi'an, P.R China
}

\begin{abstract}
Background: Outbreaks of hand-foot-and-mouth disease (HFMD) have occurred in many parts of the world, particularly in China. The disease usually has ease of communicability and a higher risk of developing severe complications leading towards death. In this study, we aim to evaluate the clinical features, laboratory examination, and treatment for serious cases of HFMD, using a retrospective analysis based on clinical data.

Methods: We report the clinical features of 22 deceased patients with confirmed diagnoses of HFMD, who presented to hospitals in the Shanxi Province of China between June 2010, and August 2012. Demographic, clinical and laboratory data from inpatients were collected.

Results: The findings of this report showed that Enterovirus 71 (EV71) is the causative agent of HFMD, particularly in male children under the age of 3. Some common symptoms including fever, rash, and malfunctioning of respiratory, circulatory, and neurologic systems were observed in all studied patients. However, circulatory failure was the most important cause of death for these patients. Furthermore, increases in the peripheral white blood cell count $\left(>15 \times 10^{9} / \mathrm{L}\right)$ and the blood sugar level $(>8.3 \mathrm{mmol} / \mathrm{L})$ are among the important reference indices for serious cases. Hence, on time provision of respiratory and circulatory supports enhance the efficiency of a comprehensive treatment strategy.

Conclusion: HFMD, characterized by high fever, convulsions, mental fatigue, vomiting and cold limbs, carries a high risk of death. Earlier identification of higher-risk infants might allow for more rapid intervention. Our findings suggest that the peripheral white blood cell count $\left(>15 \times 10^{9} / \mathrm{L}\right)$ and the blood sugar level $(>8.3 \mathrm{mmol} / \mathrm{L})$ should be considered as warning signs while, clinicians manage surges of children diagnosed with HFMD to avoid death.
\end{abstract}

Keywords: HFMD, Clinical symptoms, Complication, Treatment.

Accepted on June 20, 2018

\section{Introduction}

Hand-foot-and-mouth disease (HFMD) is a common infectious disease in children. During summer 1957, the first outbreak of HFMD was documented in Toronto. In mainland China, some reports about small-scale outbreaks of HFMD appeared in Tianjin and Shandong provinces during the 1980's. However, during 2008 an outbreak of HFMD at large scale was reported in Fuyang city of Anhui province and led to the death of 22 hospitalized patients. After this incidence, HFMD was classified as a national legal C-class infectious disease. Recently, the number of HFMD cases has been increasing rapidly in mainland China. Therefore, effective monitoring and efficient reporting strategy to deal with HFMD is still needed in some parts of the country.

It has been reported that Enteroviruses, i.e. Coxsackievirus A16 (CV-A16) and Enterovirus 71 (EV71) are the primary causative agent of HFMD. In many cases, these viruses showed clear prognosis and obvious clinical manifestations 
with a typical self-healing time of one week. However, some children especially those less than three years of age showed a typical clinical symptom with rapid onset of serious complications such as myocarditis, pulmonary edema and aseptic meningitis (Table 1). Multiple system organ failures may be secondary to these severe complications.

Between 2010 and 2013, in Xi'an city, China, about 82892 cases of HFMD have documented, and the incidence of HFMD was 251.32/100,000. A total of 10896 HFMD cases have appeared in Changan region of Xi'an city from 2010 to 2012 with an average rate of incidence is $333.33 / 100,000$. Unfortunately, a total of 22 patients of HFMD, 16 from Xi'an Children's Hospital and 6 from Center Hospital of Hanzhong, passed away during this period. However, 36 patients were successfully recovered from this disease. The current study aims to present a retrospective analysis, showing clinical features, laboratory and imaging examinations, treatment and prognosis to identify the risk factor and complications of 22 deceased and 36 recovered patients.

\section{Materials and Methods}

\section{Materials}

(1) General characteristics: Gender and age; (2) Clinical data: Hospitalization days, clinical performance, disease course; (3) Auxiliary examinations: Laboratory examination or etiological examination (EV71-DNA, EV71 serum antibody examination), blood sugar level, blood routine, blood gas analysis, electrocardiogram, imaging studies (chest X-rays, head CT and chest CT), clinical classification and treatment; (4) Prognosis.

\section{Patients}

All patients including recovered as well as deceased included in this study were diagnosed with HFMD. Notably, among 22 deceased patients, 16 were admitted to Xi'an Children's Hospital between February-August 2012, the other 6 patients were admitted to the Center Hospital of Hanzhong between June-July, 2010. The diagnosis of HFMD in the study was made by paediatricians aided by laboratory tests and neurological images. We summarized the characteristics of these 22 deceased and 36 recovered patients. Data on the patient's vital signs, physical exam findings, and routine laboratory tests were obtained through a retrospective review of the hospital records. The Ethics Committee of the Hospital has approved this study.

\section{Results}

\section{Demographic characteristic of patients}

The mean age of the patients under study was 24 months, while detailed information about gender and age distribution of the patients is presented in Figure 1. Among those patients unfortunately died includes 14 males and 8 females with the male to female ratio of $1.75: 1$. This ratio shows that the chances to acquire this disease are higher in males $(63.6 \%)$ than the females (36.4\%). Additionally, in $81.8 \%$ cases children younger than $3 \mathrm{y}$ of age became the victim of HFMD, with an average disease course and days of hospitalization was $3 \mathrm{~d}$ and $1.5 \mathrm{~d}$ respectively.

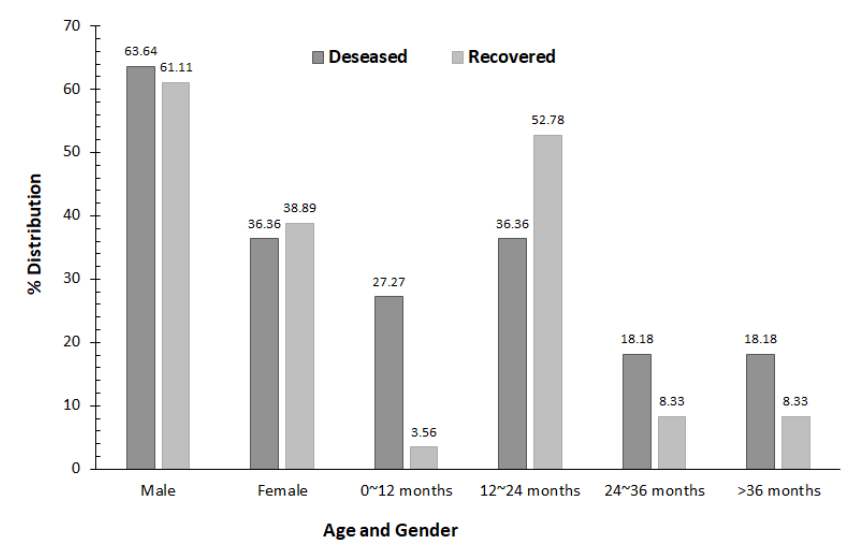

Figure 1. \% Distribution of age and gender in thirty-six recovered and twenty-two deceased HFMD patients.

\section{Clinical features}

The clinical features observed in this study are abnormalities in the nervous system, respiratory system and circulatory system, etc. All the 22 patients showed symptoms of fever with an average body temperature $38.8^{\circ} \mathrm{C}$ and rash. Furthermore, in many cases patients under observation showed neurological malfunctioning such as disturbance of consciousness $(90.9 \%)$, coma $(77.3 \%)$, convulsions $(54.5 \%)$, apathy and sluggishness $(50.0 \%)$, secretions following through throat stimulation $(40.9 \%)$, as well as respiratory symptoms including rales (100\%), and tachypnea and bradypnea (both $77.3 \%$ ) respectively. However, circulatory failure (68.2\%) was found to be the major cause of death for these patients (Figures 1 and 2).

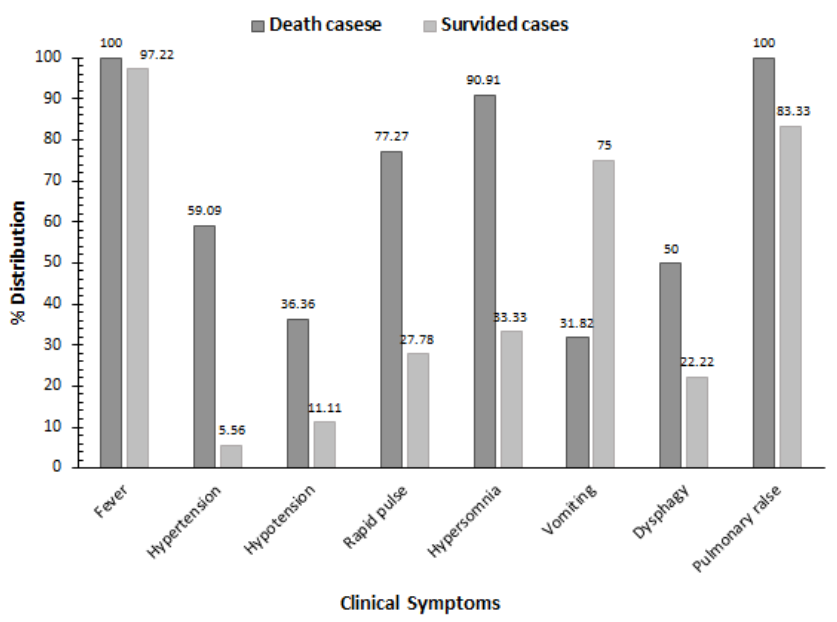

Figure 2. \% Distribution of clinical features observed in thirty-six recovered and twenty-two deceased HFMD patients.

\section{Auxiliary examination}

When hospitalized, the average leukocyte count was measured in the patients, and it was $5.2 \times 10^{9} / \mathrm{L}$ ranging from $0.8 \times 10^{9} / \mathrm{L}$ 
to $24 \times 10^{9} / \mathrm{L}$. In 5 cases the leukocyte count increased to $22.7 \%$, while in 3 cases the leukocyte count was higher than 15 $\times 109 /$ L . Contrastingly, a decrease up to $77.3 \%$ in leukocyte count was observed in 17 cases with an average lymphocyte count were $1.06 \times 10^{9} / \mathrm{L}$ ranging from $0.4 \times 10^{9} / \mathrm{L}$ to $11.07 \times$ $10^{9} / \mathrm{L}$; Moreover, examination of blood sugar revealed an increase of blood sugar in 17 cases where sugar level was higher than $8.3 \mathrm{mmol} / 1$ in 11 patients; while sugar level was normal in 4 cases, however, reduction in blood sugar was found in 1 patient. Subsequent PCR-based detection and analysis of nucleic acid in 19 patients confirmed the prevalence of Enterovirus and EV71 in 19 and 17 however, Coxsackievirus and Echoviruses in 1, 1 patients respectively.
Also, 8 patients showed an increase in myocardial enzyme $\alpha$ HBDH levels (Table 1).

Chest radiographs were performed for 19 patients. Radiographs from 13 patients showed signs of severe pneumonia, while 2 patients showed signs of pulmonary edema, and 4 patients showed signs of both pneumonia and pulmonary edema. All of the patients underwent electrocardiograms. Eleven patients showed nodal tachycardia, while seven showed segment depression (ST), three showed sinus bradycardias and one patient appeared ventricular arrhythmia when he was in rescue (Table 1).

Table 1. Physicochemical tests and PCR based identification of viruses in twenty-two deceased/death and 36 recurred patients of HFMD.

\begin{tabular}{|c|c|c|c|c|}
\hline \multirow{2}{*}{ Type of examination } & \multicolumn{2}{|c|}{ Deceased/dead patients } & \multicolumn{2}{|l|}{ Recovered patients } \\
\hline & \# of dead patients & Prevalence (\%) & \# of survived patients & Prevalence (\%) \\
\hline WBC $\uparrow^{a}$ & 5 & 22.73 & 23 & 63.88 \\
\hline Blood sugar $\uparrow^{b}$ & 17 & 77.27 & 24 & 66.67 \\
\hline Chest radiograph and pulmonary edema & 6 & 27.7 & 12 & 33.33 \\
\hline \multicolumn{5}{|c|}{ Virus identification in 19 and 36 patients who died and survived respectively } \\
\hline Echovirus 71 (EV71) & 17 & 89.47 & 34 & 94.44 \\
\hline Coxsackievirus & 1 & 5.26 & 1 & 2.78 \\
\hline Echovirus & 1 & 5.26 & ND & - \\
\hline Enterovirus others & ND & - & 1 & 2.78 \\
\hline
\end{tabular}

aBC>12 × 109/L; bBlood sugar $>6.1 \mathrm{mmol} / \mathrm{L}$; ND: Not Determined.

\section{Discussion}

Hand-foot-and-mouth-disease (HFMD) represents complications of infectious disease caused by a variety of Enteroviruses, with various clinical manifestations. The primary population infected with HFMD is young children, especially those less than $3 \mathrm{y}$ old. Clinical manifestations of most cases are mild, with typical symptoms being fever and rash, which can be found on the hand, foot, and hip, etc. In a few cases, severe damage occurs to the nervous system, respiratory system, and circulatory system, causing encephalitis, flaccid paresis, myocarditis, pulmonary edema and other complications leading to the death of the patient. By analyzing the clinical features of 22 cases of deceased children with a diagnosis of HFMD. We summarized some highly significant features of the children who have rapidly developed severe conditions (Table 2). The outcomes of this work help clinicians to closely follow changes in the clinical condition to carry out necessary laboratory examinations and to determine the best treatment plan to reduce the rate of mortality. We found that the rate of onset of HFMD is higher in male children as compared to females. Further, the rater of mortality in children younger than $3 \mathrm{y}$ was $81.8 \%$, while this rate was $63.7 \%$ in children younger than $2 \mathrm{y}$. These findings make us put more emphasis on prevention and monitoring of HFMD in children younger than $3 \mathrm{y}$ of age. Etiological examination showed that in most of the cases Enterovirus 71 was found to be the causative agent of HFMD and these findings are in-line with previously documented reports [1-3].

Table 2. Complications observed in twenty-two deceased/death and 36 recurred patients of HFMD.

\begin{tabular}{lcclll}
\hline Complications found in deceased/dead patients & \multicolumn{2}{l}{ Complications found in recovered patients } \\
\hline Complications & $\begin{array}{l}\text { \# of dead } \\
\text { patients }\end{array}$ & Prevalence (\%) & Complications & \# of recovered patients & Prevalence (\%) \\
\hline Circulatory failure & 15 & 68.18 & $\begin{array}{l}\text { Brainstem encephalitis together } \\
\text { with pulmonary edema }\end{array}$ & 18
\end{tabular}




\begin{tabular}{lcclcc}
\hline NFE & 8 & 36.36 & Stress ulcer & 11 & 30.56 \\
\hline Respiratory failure & 2 & 9.09 & $\begin{array}{l}\text { Encephalitis together } \\
\text { pulmonary edema }\end{array}$ & with 10 & 27.78 \\
\hline Septic shock & 1 & 4.55 & Myocardial damage & 10 & 27.78 \\
\hline MODS & 1 & 4.55 & & & \\
\hline NFE & 8 & 36.36 & & & \\
\hline Septic shock & 1 & 4.55 & & & \\
\hline
\end{tabular}

The diagnosis and treatment guidelines for HFMD formulated by Ministry of Health of the People's Republic of China-2010 state that increases in the peripheral white blood cell count (15 $\left.\times 10^{9} / \mathrm{L}\right)$ and the blood sugar level $(>8.3 \mathrm{mmol} / \mathrm{L})$ are among the critical reference indices for severe cases. In this study, patients with increased white blood cells counts and blood sugar levels accounted for $22.7 \%$ and $77.3 \%$ of cases, respectively [4].

According to the clinical process, HFMD can be divided into five periods: (1) The skin eruption period; (2) The nervous system involvement period; (3) The early period of cardiopulmonary failure; (4) The cardiopulmonary failure period; (5) The recovery period [5,6]. The key to proper diagnosis and treatment is to identify the second and third periods in an accurate and timely fashion.

The following signs implied that the patients might develop serious complications: (1) Sustained high fever, with axilla temperature $>39^{\circ} \mathrm{C}$ and failure of conventional methods to lower the temperature; (2) Nervous symptoms, including mental fatigue, vomiting, restlessness, convulsions; (3) Abnormal breathing, including tachypnea, bradypnea, or discordant breathing rhythms; If breathing rate exceeds 30 breaths/min (accounting for age), the patient may have neurogenic pulmonary edema; (4) Abnormal circulation, including cold sweats, cold extremities, tachycardia $(>140-150$ beats per min, accounting for age), elevated blood pressure and delayed capillary refill time; (5) Increases in the peripheral white blood cell count $\left(>15 \times 10^{9} / \mathrm{L}\right)$ and blood sugar levels $(>8.3 \mathrm{mmol} / \mathrm{L})$. In cases of suspected nervous system involvement, the cerebrospinal fluid examination should be timely $[7,8]$.

The following issues are important with regards to treatment $[9,10]$ : For typical cases, symptomatic treatment should be adopted [11,12]. However, clinicians should additionally monitor the patient's blood pressure, heart rate, and breathing and blood sugar levels and perform a neurological examination to improve the understanding of severe cases. Mortality in severe cases is always caused by severe complications [13]. In this study of 22 cases of mortality, $68.2 \%$ cases suffered from circulatory failure, $36.4 \%$ from NFE and $9.1 \%$ from respiratory failure.

Clinicians should master the proper treatment of severe complications to prevent mortality. First, restriction of fluid intake should be taken into consideration for severe cases of cerebral edema, pulmonary edema or cardiac failure. The commonly used treatment for dehydration is a $20 \%$ mannitol solution $(0.5 \sim 1.0 \mathrm{~g} / \mathrm{kg})$ given every $4-8 \mathrm{~h}$ over $20-30 \mathrm{~min}$ via rapid intravenous injection. Second, the application of intravenous immunoglobulin (IVIG) may play a role in treatment for patients presenting with restlessness, frequent convulsions, acute limb paralysis, rapid increases in the breathing rate, cold sweats, cold extremities, and rapid increases in the heart rate $[14,15]$. The treatment should be given at a dose of $1.0 \mathrm{~g} /(\mathrm{kg} . \mathrm{d}$ ) (maintenance for $2 \mathrm{~d}$ ). Third, methylprednisolone pulse therapy may relieve the cerebral edema and pulmonary edema caused by EV71 [16]. This therapy should be given at a dose of 3-5 $\mathrm{mg} /(\mathrm{kg} . \mathrm{d})$ and halted as soon as the patient reaches a stable condition (Table 3).

Table 3. Treatments given to 22 deceased/death and 36 recurred patients of HFMD.

\begin{tabular}{|c|c|c|c|c|}
\hline \multirow{2}{*}{ Treatments include } & \multicolumn{2}{|c|}{$\begin{array}{l}\text { Deceased/dead } \\
\text { patients }\end{array}$} & \multicolumn{2}{|c|}{ Recovered patients } \\
\hline & $\begin{array}{l}\# \text { of } \\
\text { dead } \\
\text { patients }\end{array}$ & $\begin{array}{l}\text { Recovery } \\
\text { (\%) }\end{array}$ & $\begin{array}{l}\# \text { of } \\
\text { survived } \\
\text { patients }\end{array}$ & $\begin{array}{l}\text { Recover } \\
\text { y (\%) }\end{array}$ \\
\hline $\begin{array}{l}\text { Methylprednisolone pulse } \\
\text { therapy }\end{array}$ & 21 & 95.45 & 36 & 100 \\
\hline IVIG & 17 & 77.27 & 36 & 100 \\
\hline Mannitol & 15 & 68.18 & 36 & 100 \\
\hline Anti-shock measures & 20 & 90.91 & 36 & 100 \\
\hline Anti-infection measures & 21 & 95.45 & 36 & 100 \\
\hline Nical ventilation & 21 & 95.45 & 36 & 100 \\
\hline
\end{tabular}

Also, mechanical ventilation should be quickly initiated when the patient presents with discordant breathing rhythms, bloody airway secretions, rapid onset of rales, and a frequent conclusion with deep coma, pallor, cyanosis and hypotension. Mechanical ventilation should not be withdrawn prematurely. The indications for withdrawal of mechanical ventilation are as follows: (1) Return of normal spontaneous breathing and cough reflex; (2) The oxygenation index $\left(\mathrm{OI}=\mathrm{PaO}_{2} / \mathrm{FiO}_{2} \times 100\right)>300$ $\mathrm{mmHg}$ with chest X-ray improvement; (3) Return of consciousness; (4) Stable circulation; (5) No life-threatening complications present.

When the patient reaches a stable condition, their swallowing capabilities may remain compromised. It may be necessary to 
continue a special diet and wean mechanical ventilation slowly. Clinicians should also keep a high suspicion for respiratory infection [17-22].

In summary, in our clinical experience patients with serious cases of HFMD should be admitted to the PICU for close observation following diagnosis to receive symptomatic treatment and to avoid serious complications. A modest dose of mannitol should be given early to drop intracranial pressure when the patient presents with neurological symptoms. For new cases, including cases of cardiopulmonary failure and pulmonary edema, the use of intravenous immunoglobulin and hormone therapy is necessary. In these severe cases, clinicians must provide comprehensive treatment including respiratory and circulatory support to effectively treat the pulmonary edema period and reduce mortality.

\section{Conflicts of Interest}

Authors declare no conflict of interest.

\section{References}

1. Lee M-H, Huang LM, Wong W-W, Wu T-Z, Chiu T-F, Chang L-Y. Molecular diagnosis and clinical presentations of enteroviral infections in Taipei during the 2008 epidemic. J Microbiol Immunol Infect 2011; 44: 178-183.

2. Zhang ZJ, Cui CY, Shi LH. Analysis on Epidemiological Characteristics of Hand, Foot and Mouth Disease in Xi'an, 2010-2013. Mod Preventive Med 2015; 7: 1162-1167.

3. Mao X, Wang XL. The epidemiological analysis of hand, foot and mouth disease in Changan district of Xi'an, 2010-2012. Spec Health 2014; 8: 613.

4. Sawyer MH. Enterovirus infections: diagnosis and treatment. Seminars in pediatric infectious diseases. Elsevier 2002; 13: 40-47.

5. Redington JJ, Tyler KL. Viral infections of the nervous system, 2002: update on diagnosis and treatment. Arch Neurol 2002; 59: 712-718.

6. Zeng M, El Khatib NF, Tu S, Ren P, Xu S, Zhu Q. Seroepidemiology of Enterovirus 71 infection prior to the 2011 season in children in Shanghai. J Clin Virol 2012; 53: 285-289.

7. Ang L-W, Phoon M-C, Wu Y, Cutter J, James L, Chow VT. The changing seroepidemiology of enterovirus 71 infection among children and adolescents in Singapore. BMC Infect Dis 2011; 11: 270.

8. Zhang J, Jun Ling S, Chang Z, Zhang W, Zi Jun W, Zi Jian F. Characterization of hand, foot, and mouth disease in China between 2008 and 2009. Biomed Environ Sci 2011; 24: 214-221.

9. Organization WH. A guide to clinical management and public health response for hand, foot and mouth disease (HFMD). WHO Regional Office for the Western Pacific, Manila 2011.

10. National Health and Family Planning Commission. According to the guidelines of the National Health and Family Planning Commission (NHFPC) of the People's
Republic of China for diagnosis and treatment of HFMD. NHFPC 2010

11. Huang M-C, Wang S-M, Hsu Y-W, Lin H-C, Chi C-Y, Liu C-C. Long-term cognitive and motor deficits after enterovirus 71 brainstem encephalitis in children. Pediatrics 2006; 118: 1785-1788.

12. Li W, Teng G, Tong H, Jiao Y, Zhang T, Chen H. Study on risk factors for severe hand, foot and mouth disease in China. PloS One 2014; 9: e87603.

13. Kim SJ, Kim J-H, Kang J-H, Kim DS, Kim KH, Kim KH. Risk factors for neurologic complications of hand, foot and mouth disease in the Republic of Korea, 2009. J. Korean Med Sci 2013; 28: 120-127.

14. Tsao K-C, Chang P-Y, Ning H-C, Sun C-F, Lin T-Y, Chang L-Y. Use of molecular assay in diagnosis of hand, foot and mouth disease caused by enterovirus 71 or coxsackievirus A 16. J Virol Methods 2002; 102: 9-14.

15. Stalkup JR, Chilukuri S. Enterovirus infections: a review of clinical presentation, diagnosis, and treatment. Dermatol Clin 2002; 20: 217-223.

16. Thomas I, Janniger C. Hand, foot, and mouth disease. Cutis 1993; 52: 265.

17. Bendig J, Fleming D. Epidemiological, virological, and clinical features of an epidemic of hand, foot, and mouth disease in England and Wales. Communic Dis Rep CDR Rev 1996; 6: 81-86.

18. Wu T-N, Tsai S-F, Li S-F, Lee T-F, Huang T-M, Wang ML. Sentinel surveillance for enterovirus 71, Taiwan, 1998. Emerging Infect Dis 1999; 5: 458.

19. Wang S-M, Lei H-Y, Huang M-C, Su L-Y, Lin H-C, Yu C$\mathrm{K}$. Modulation of cytokine production by intravenous immunoglobulin in patients with enterovirus 71associated brainstem encephalitis. J Clin Virol 2006; 37 : 47-52.

20. Guang-Chao S, Si-Da Y, Jian-Ping T, Hua-Song Z. Analysis of peripheral blood lymphocyte subpopulations in children with severe and critical hand, foot and mouse disease. Chin J Evidence-Based Pediatr 2010; 4: 005.

21. Nolan M, Craig M, Lahra M, Rawlinson W, Prager P, Williams G. Survival after pulmonary edema due to enterovirus 71 encephalitis. Neurology 2003; 60: 1651-1656.

22. Jiao FY, Zhang YY, Li KX. Advances in treatment of hand foot and mouth disease. China Med Pharm 2012; 219: 38-40.

\section{*Correspondence to}

Fiaz Ahmad

Key Laboratory for Space Bioscience and Biotechnology

School of Life Sciences

North-Western Polytechnical University

Shaanxi

P.R China 